\title{
Evaluating the Effects of Traditional Persian Music on Nonlinear Parameters of HRV
}

\author{
Bahareh Khodabakhshian $^{1}$, Sadaf Moharreri ${ }^{1}$, Saman Parvaneh ${ }^{2 *}$ \\ ${ }^{1}$ Islamic Azad University, Khomeini Shahr Branch, Isfahan, Iran \\ ${ }^{2}$ Islamic Azad University, Science and Research Branch, Tehran, Iran \\ ${ }^{*}$ Currently with Philips Research North America, Cambridge, MA, USA
}

\begin{abstract}
Music has the power to evoke particular emotional states. In this research, the impact of three types of traditional Persian music (happy, peaceful, and sad) on nonlinear parameters for heart rate variability (HRV) analysis is studied. After extracting RR intervals from $E C G$, the nonlinear parameters were obtained. The parameters include normal descriptors of Poincare plot (SD1 and SD2), Global Occurrence Matrix (GOM), and Co-occurrence Matrix (COM) parameters which demonstrate the dynamic in the Poincare plot. The extracted features in three groups of music stimuli were compared with the controls and then k-nearest neighbor classifier used to distinguish different emotions induced by the different music. The results show that the GOM and COM features were significantly different between different emotions induced by music stimuli. Promising results on emotion classification (accuracy of 90\%) in response to music stimuli highlight the power of nonlinear analysis of HRV in emotion assessment application.
\end{abstract}

\section{Introduction}

Music has the power to evoke particular emotional states and can affect mood, for example, by decreasing the mental stress [1]. Music can induce different emotions that can be captured through physiological response, such as heart rate and heart rate variability (HRV) [2]. Irrespective of music application for therapeutic purposes, little is known about how music can impact physiological parameters [4].

HRV analysis in nonlinear domain is used for emotion recognition while listening to music intended to stimulate certain emotions [5]. In this study, three emotional classes, including happy, peaceful, and sad were induced by three types of traditional Persian music. Feature extracted from Poincare plot of RR intervals were extracted in this study, and induced emotions were detected using K-nearest neighbor classifier.

\section{Method and Data}

Outline of the proposed methodology in this study is shown in Figure 1.

\subsection{Music Stimuli and Data Collection}

ECG of 22 female volunteers without diagnosed cardiac arrhythmia (Age: $22.72 \pm 2.52$ ) was recorded in a control (silence) condition and while listening to three types of traditional Persian music that evoke certain emotions (happy, peaceful, and sad). ECGs have been recorded from lead П. Powerlab system (Model: ML4866) was used for data acquisition. The stimulation protocol is represented in Figure 2. The laboratory conditions (e.g., temperature and light) were kept fixed for all participants as far as possible. The mean temperature was about $24^{\circ} \mathrm{C}$, and subjects were asked to close their eyes during data acquisition. A bandpass filter was used for removing power line interference, and a single stage of median filtering was used for eliminating baseline wander (Figure 3). After QRS detection, RR intervals were extracted for HRV analysis (Figure 4).

\subsection{SAM Test for Labeling Data}

Labeling emotion perceived by users during an experiment by direct questioning is prone to bias as participants might not reflect their real emotion. To solve this problem, Self- Assessment Manikin (SAM) has been used, which is more reliable to capture subjects' emotions $[8,9]$.

SAM test contains three dimensions: arousal, valence, and dominance. Five manikins are used graphically in each dimension to determine the levels of arousal, valence, and dominance (Figure 5). The numeric value of these figures for each level, respectively from left to right are $-2,-1,0$, 1 , and 2. In this study, each participant completed the SAM test after each stimulus, and the results were used to 


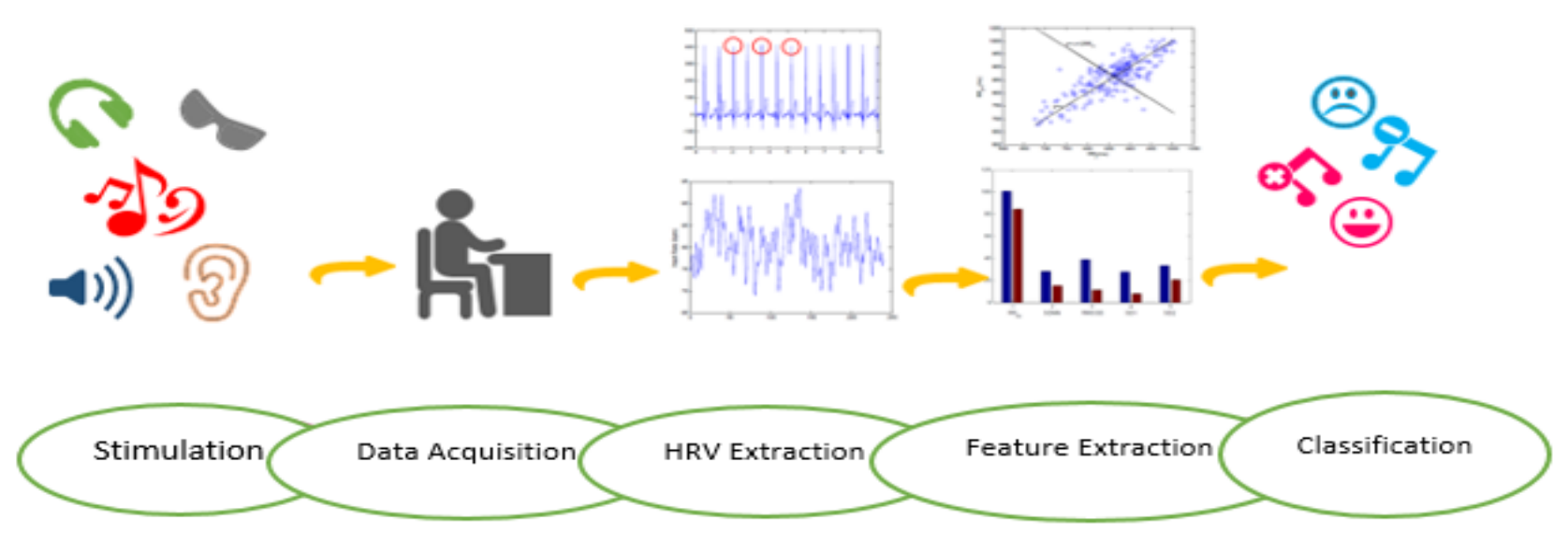

Figure 1. Outline of proposed methodology.

confirm the impact of three types of Persian music on emotions.

\subsection{Feature Extraction}

\subsubsection{Poincare Plot}

The Poincare plot is a geometrical representation of $\mathrm{RR}$ time series $\left(R R=R R_{1}, R R_{2}, \ldots, R R_{n}\right)$ that were proposed for non-linear analysis of HRV. Poincare plot indicates pairs as $p_{i}=\left(x_{i}, y_{i}\right)$ with $i=1,2,3, \ldots$ in which:

$x=\left\{x_{1}, x_{2}, \ldots, x_{n}\right\}=\left\{R R_{1} \cdot R R_{2}, \ldots, R R_{n-1}\right\}$

$y=\left\{y_{1}, y_{2}, \ldots, y_{n}\right\}=\left\{R R_{2} . R R_{3}, \ldots, R R_{n}\right\}$

where $\mathrm{n}$ is the number of data points [8].

Standard descriptors of Poincare plot are SD1 and SD2. $\mathrm{SD} 1$ is the standard deviation on the line perpendicular to the identity line $(y=-x)$, and SD2 is defined as the standard deviation on the identity line $(\mathrm{y}=\mathrm{x})$ [9].

$$
\begin{array}{ll}
S D 1=\sqrt{\operatorname{Var}(d 1)} & S D 2=\sqrt{\operatorname{Var}(d 2)} \\
d 1=\frac{x-y}{\sqrt{2}} & d 2=\frac{x+y}{\sqrt{2}}
\end{array}
$$

\subsubsection{Global Occurrence Matrix (GOM) and Co-occurrence Matrix (COM)}

Global occurrence matrix specifies the distribution of points in Poincare plot relative to the identity line $(y=x)$ [10]. The points' position relative to the identity line in Poincare plot have different meanings. The points on this line correspond to equal consecutive $R R$ intervals, the points above and below this line corresponds to decreasing and increasing heart rate. In $G O M$, the points in Poincare plot are partitioned into three regions:

- Points which are above the identity line (A);

- Points which are on the identity line $(\mathrm{O})$;

- Points which are below the identity line (B).

In global method, a $3 \times 1$ vector of GOM was constructed by counting number of points in each region [11].

$G O M=\left[\begin{array}{lll}N_{A} & N_{O} & N_{B}\end{array}\right]$

Also, every two consecutive points $P_{i}$ and $P_{i+l}$ in Poincare plot create nine features for COM based on position of these two points relative to each other and identity line [10]:

$$
C O M=\left[\begin{array}{ccc}
A A & A O & A B \\
O A & O O & O B \\
B A & B O & B B
\end{array}\right]
$$

Where $\operatorname{Var}(d)$ is the variance of $d$. 


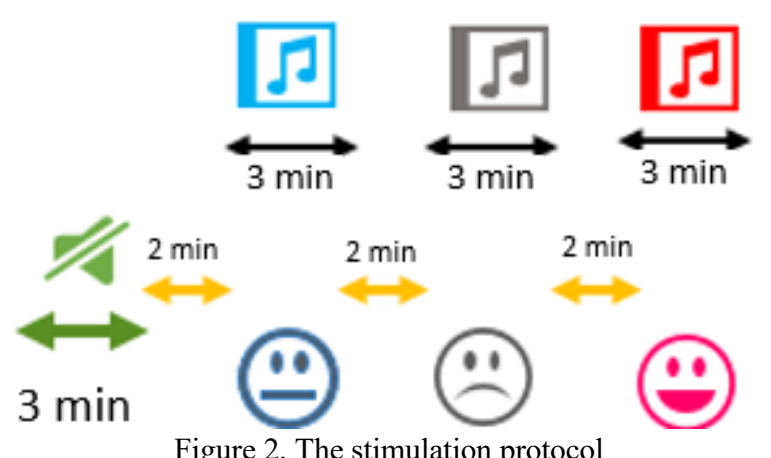

Figure 2. The stimulation protocol
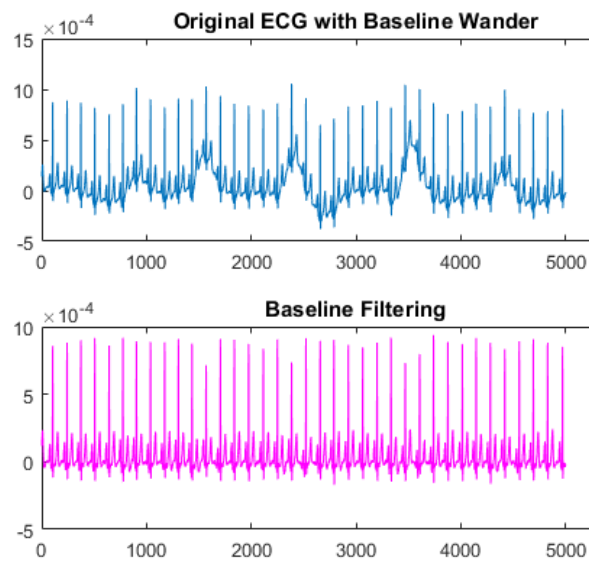

Figure 3. A typical ECG before and after baseline removal
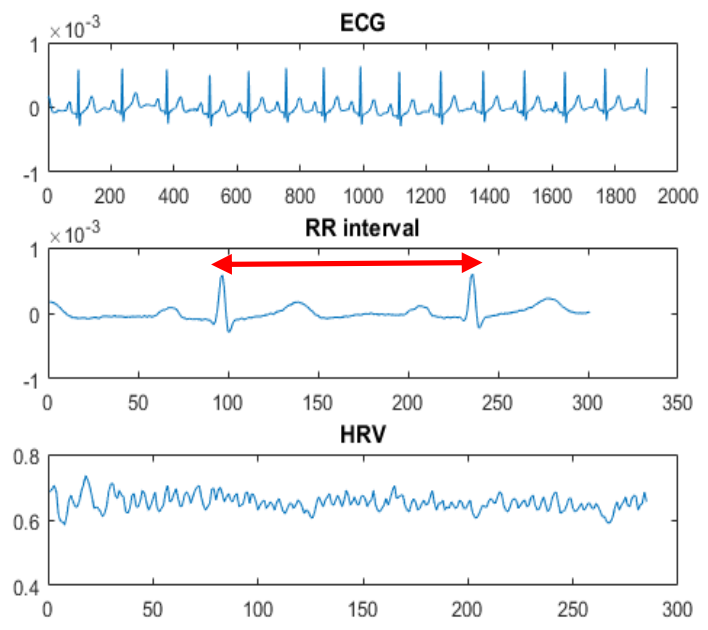

Figure 4. Extraction of RR series from ECG. The top figure shows a typical ECG signal recorded in this study, the second plot shows the extraction of a single RR interval, and the third one represents the extraction of the RR Intervals.
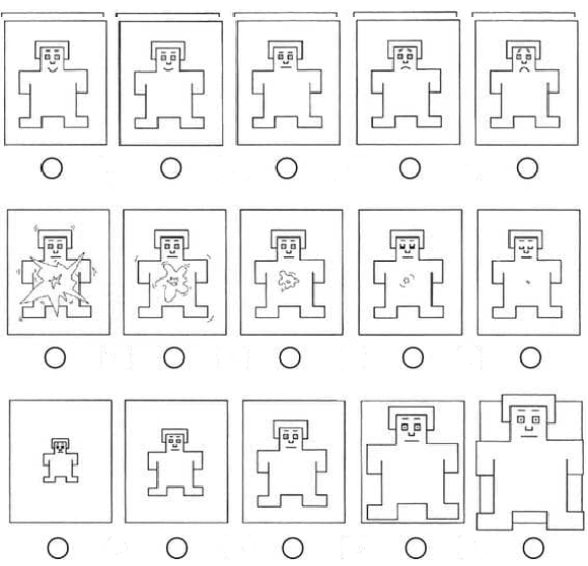

Figure 5. Self-assessment Manikin test.

\subsection{Statistical Analysis and Classifier}

First Kruskal-Wallis test, non-parametric version of ANOVA, was performed to compare extracted features between control and different music groups. Level of statistical significance set as $P$-value equal to 0.05 . Then K-Nearest Neighbor (KNN) with K equal to 3 (minimum error as shown in Figure 6) was used to classify these groups which was trained on $70 \%$ data as a training set and $30 \%$ as a test set.

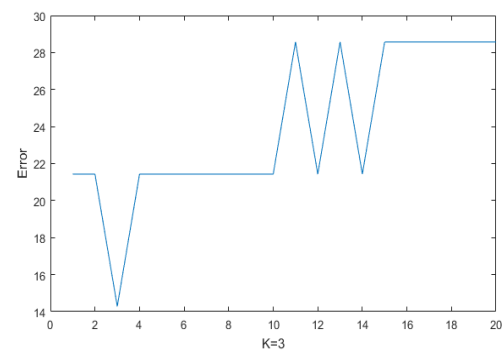

Figure 6. Error rate versus number of neighbors in $\mathrm{KNN}$

\section{Results}

Mean and standard deviation of extracted features for control and different music groups are reported in Table 1. $P$-value for comparison of standard descriptors of Poincare plot, GOM features, and COM features could be found in Table 2. Classification results are shown in Table 3.

\section{Discussion}

In this study, three emotional classes, including happy, peaceful, and sad were induced by three kinds of traditional Persian music. Induced emotions were identified using heart response and KNN classifier. The results show that the traditional Persian music can induce different feelings 
that could be detected using HRV analysis. However, the impact of culture on induced emotion and the associated physiological response needs to be explored in future studies.

Table 1. Mean and standard deviation of features in control and three music groups.

\begin{tabular}{|c|c|c|c|c|}
\hline 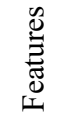 & 晜 & 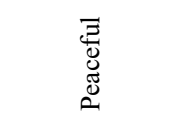 & $\begin{array}{l}\overrightarrow{\tilde{E}} \\
\tilde{n}\end{array}$ & $\begin{array}{l}\text { ڤे } \\
\text { 心्ञ } \\
\text { İ }\end{array}$ \\
\hline SD1 & $0.02 \pm 0.01$ & $0.02 \pm 0.01$ & $0.02 \pm 0.01$ & $0.02 \pm 0.01$ \\
\hline SD2 & $0.05 \pm 0.02$ & $0.05 \pm 0.02$ & $0.05 \pm 0.01$ & $0.05 \pm 0.02$ \\
\hline $\mathrm{N}_{\mathrm{A}}$ & $97.55 \pm 13.00$ & $93.55 \pm 14.45$ & $113.14 \pm 17.54$ & $102.2 \pm 14.6$ \\
\hline $\mathrm{N}_{\mathrm{O}}$ & $17.73 \pm 16.59$ & $20.18 \pm 16.33$ & $28.00 \pm 24.17$ & $22.45 \pm 18.3$ \\
\hline $\mathrm{N}_{\mathrm{B}}$ & $92.73 \pm 6.30$ & $89.27 \pm 5.26$ & $110.86 \pm 9.40$ & $99.27 \pm 7.71$ \\
\hline $\mathrm{AA}$ & $49.59 \pm 12.44$ & $46.14 \pm 13.85$ & $57.14 \pm 14.41$ & $48.41 \pm 12.4$ \\
\hline $\mathrm{AO}$ & $6.77 \pm 4.88$ & $6.82 \pm 4.89$ & $9.64 \pm 7.25$ & $8.73 \pm 6.40$ \\
\hline $\mathrm{AB}$ & $40.82 \pm 9.90$ & $40.18 \pm 9.74$ & $45.95 \pm 12.64$ & $44.64 \pm 11.3$ \\
\hline $\mathrm{OA}$ & $7.73 \pm 6.66$ & $9.05 \pm 7.09$ & $11.50 \pm 8.66$ & $8.77 \pm 5.74$ \\
\hline $\mathrm{OO}$ & $2.91 \pm 4.72$ & $2.86 \pm 3.72$ & $5.55 \pm 7.39$ & $3.55 \pm 4.69$ \\
\hline $\mathrm{OB}$ & $6.95 \pm 6.15$ & $8.18 \pm 6.36$ & $10.86 \pm 9.29$ & $10.05 \pm 8.87$ \\
\hline $\mathrm{BA}$ & $39.64 \pm 11.79$ & $37.64 \pm 11.66$ & $44.14 \pm 13.25$ & $44.55 \pm 11.0$ \\
\hline $\mathrm{BO}$ & $8.00 \pm 8.06$ & $10.41 \pm 8.76$ & $12.59 \pm 10.37$ & $10.14 \pm 7.95$ \\
\hline $\mathrm{BB}$ & $44.59 \pm 8.34$ & $40.73 \pm 9.21$ & $53.64 \pm 10.34$ & $44.18 \pm 8.49$ \\
\hline
\end{tabular}

Table 2. P-value to compare different groups of music and control.

\begin{tabular}{|c|c|c|c|c|c|c|}
\hline 总 & 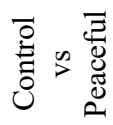 & 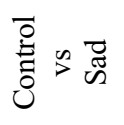 & 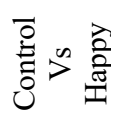 & 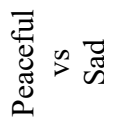 & 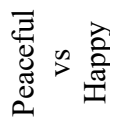 & 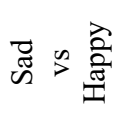 \\
\hline SD1 & 0.481 & 0.291 & 0.348 & 0.814 & 0.851 & 0.851 \\
\hline SD2 & 0.796 & 0.963 & 0.778 & 0.673 & 0.814 & 0.944 \\
\hline $\mathrm{N}_{\mathrm{A}}$ & 0.391 & 0.003 & 0.181 & $<0.01$ & 0.036 & 0.028 \\
\hline $\mathrm{N}_{\mathrm{O}}$ & 0.533 & 0.192 & 0.411 & 0.404 & 0.733 & 0.549 \\
\hline $\mathrm{N}_{\mathrm{B}}$ & 0.026 & $<0.01$ & 0.018 & $<0.01$ & $<0.01$ & $<0.01$ \\
\hline $\mathrm{AA}$ & 0.418 & 0.100 & 0.814 & 0.027 & 0.581 & 0.038 \\
\hline $\mathrm{AO}$ & 0.972 & 0.258 & 0.377 & 0.212 & 0.353 & 0.769 \\
\hline $\mathrm{AB}$ & 0.459 & 0.135 & 0.139 & 0.048 & 0.088 & 0.681 \\
\hline $\mathrm{OA}$ & 0.525 & 0.221 & 0.488 & 0.359 & 0.944 & 0.323 \\
\hline $\mathrm{OO}$ & 0.609 & 0.273 & 0.574 & 0.499 & 0.875 & 0.546 \\
\hline $\mathrm{OB}$ & 0.473 & 0.203 & 0.305 & 0.480 & 0.680 & 0.851 \\
\hline $\mathrm{BA}$ & 0.347 & 0.301 & 0.176 & 0.063 & 0.032 & 0.944 \\
\hline $\mathrm{BO}$ & 0.323 & 0.204 & 0.284 & 0.733 & 0.953 & 0.488 \\
\hline $\mathrm{BB}$ & 0.152 & 0.008 & 0.851 & $<0.01$ & 0.300 & 0.004 \\
\hline
\end{tabular}

Table 3. Classification results with KNN.

\begin{tabular}{cccc}
\hline Emotions & Accuracy & Sensitivity & Specificity \\
\hline Peaceful & 90 & 90 & 100 \\
Sad & 90 & 90 & 90 \\
Happy & 90 & 70 & 100 \\
\hline
\end{tabular}

\section{Acknowledgments}

The authors would like to give their special thanks to Mrs. Karimzadeh for the composition of the music and the volunteers for participating in the study.

\section{References}

[1] B. Abedi, A. Abbasi, A. Goshvarpour, H. T. Khosroshai, and E. Javanshir, "The effect of traditional Persian music on the cardiac functioning of young Iranian women," Indian Heart J., vol. 69, no. 4, pp. 491-498, 2017.

[2] Y. Z. Tan, S. Ozdemir, A. Temiz, and F. Celik, "The effect of relaxing music on heart rate and heart rate variability during ECG GATED-myocardial perfusion scintigraphy," Complement. Ther. Clin. Pract., vol. 21, no. 2, pp. 137-140, 2015.

[3] N. J. Dabanloo, S. Moharreri, S. Parvaneh, and A. M. Nasrabadi, "New representation of heart rate and evaluation of extracted geometric features," in Computing in Cardiology, 2010, 2010, pp. 77-80.

[4] M. Orini, R. Bailón, R. Enk, S. Koelsch, L. Mainardi, and P. Laguna, "A method for continuously assessing the autonomic response to music-induced emotions through HRV analysis," Med. Biol. Eng. Comput., vol. 48, no. 5, pp. 423-433, 2010.

[5] M. Nardelli, G. Valenza, A. Greco, A. Lanata, and E. P. Scilingo, "Recognizing emotions induced by affective sounds through heart rate variability," IEEE Trans. Affect. Comput., vol. 6, no. 4, pp. 385-394, 2015.

[6] S. Rezaei, S. Moharreri, S. Ghiasi, and S. Parvaneh, "Emotion recognition using parabolic phase space mapping for heart rate variability analysis," in 2017 Computing in Cardiology (CinC), 2017, pp. 1-4.

[7] K.-H. Choi, J. Kim, O. S. Kwon, M. J. Kim, Y. H. Ryu, and J.-E. Park, "Is heart rate variability (HRV) an adequate tool for evaluating human emotions?-A focus on the use of the International Affective Picture System (IAPS)," Psychiatry Res., vol. 251, pp. 192-196, 2017.

[8] J. Piskorski and P. Guzik, "Geometry of the Poincaré plot of RR intervals and its asymmetry in healthy adults," Physiol. Meas., vol. 28, no. 3, p. 287, 2007.

[9] J. Piskorski and P. Guzik, "Filtering poincare plots," Comput. methods Sci. Technol., vol. 11, no. 1, pp. 39-48, 2005.

[10] S. Moharreri, S. Parvaneh, N. J. Dabanloo, and A. M. Nasrabadi, "Utilizing occurrence sequence of Heart Rate's phase space points in order to discriminate heart Arrhythmia," in 2010 17th Iranian Conference of Biomedical Engineering (ICBME), 2010, pp. 1-4.

[11] S. Rezaei, S. Moharreri, S. Ghiasi, and S. Parvaneh, "Diagnosis of Sleep Apnea by Evaluating Points Distribution in Poincare Plot of RR Intervals," no. 1, pp. 3-6.

Address for correspondence.

Saman Parvaneh

2 Canal Park, 3rd floor, Cambridge, MA 02141

parvaneh@ieee.org 